\title{
Numerical simulations of radial heating in the Galaxy
}

\author{
J. Hänninen and Chris Flynn
}

\author{
Tuorla Observatory, University of Turku, Väisäläntie 20, 21500 Piikkiö, Finland
}

Received 14 July 2003 / Accepted 1 April 2004

\begin{abstract}
It has long been known that older stellar populations have higher velocity dispersion in the Solar neighbourhood than the younger ones. This has been interpreted as a 'heating' of the stellar disk as a function of time. In a previous paper we examined this heating via numerical simulations. We concentrated on the heating due to the perturbations by giant molecular clouds (GMCs) and putative massive compact dark halo objects in the Solar neighbourhood. With an appropriate combination of massive halo objects and GMCs we could fit the observed heating of the stellar disk in the Solar neighbourhood within observational accuracy.

In order to further explore the implications of the heating of the stellar disk by putative massive dark halo objects we have now examined via numerical simulations how the velocity dispersion evolves globally - not just in the Solar neighbourhood. We compare our results to the observational data by Lewis \& Freeman (1989). We find that the stellar disk heating due to massive halo objects of mass $10^{6} M_{\odot}$ ( $80 \%$ of the dark halo would be comprised of these objects) and the presently observed molecular gas distribution is not inconsistent with the observations.
\end{abstract}

Key words. Galaxy: kinematics and dynamics - Galaxy: evolution - methods: numerical

\section{Introduction}

It is well-known that random motions of stars in the Galactic disk increase with stellar age. There are four main approaches to explain this heating effect, known as the disk age-velocityrelation (hereafter AVR). The first mechanism explains the observed heating to be result of gravitational perturbations by giant molecular clouds (GMCs; Spitzer \& Schwarzschild 1951, 1953). The second mechanism utilizes transient spiral arms which are supposed to act as scattering agents (Barbanis \& Woltjer 1967). Thirdly, the massive compact halo objects (like black holes (BHs) or dark clusters) could heat up the stellar disk (Lacey \& Ostriker 1985; Carr \& Lacey 1987). Fourthly, the possibility of infall of satellite galaxies has been proposed to explain the observed AVR (Velazquez \& White 1999).

All of the above mentioned heating mechanisms have their flaws: there are insufficient GMCs in the Solar neighbourhood to explain the observed amount of the heating (Hänninen \& Flynn 2002); the heating caused by the transient spiral arms is not efficient enough in the vertical direction (Carlberg 1987); massive dark halo objects have to be very massive and compact in order to heat the stellar disk by the observed amount; infalling satellite galaxies may induce too abrupt heating peaks. Combinations of some of these mechanisms have also been proposed: the most popular being the combination of GMCs and transient spiral arms. In this model the transient spiral arms would be the main source of heating and GMCs would redirect some of the random velocities in the vertical direction

Send offprint requests to: J. Hänninen,

e-mail: jyrki.hanninen@utu.fi
(Jenkins \& Binney 1990; Jenkins 1992). However, only empirical parametrization has been so far possible in this model, because we do not have adequate information concerning e.g. the strengths, growth rates, or duty cycles of spiral features. Furthermore, it was demonstrated by Hänninen \& Flynn (2002) that the perturbations by GMCs on stellar orbits create a flatter velocity ellipsoid than earlier thought, resulting in less effective redirection of random kinetic energy in the vertical direction.

As discussed in Hänninen \& Flynn (2002, Paper I hereafer), the observational data indicate that the stellar velocity dispersion has increased from $10-20 \mathrm{~km} \mathrm{~s}^{-1}$ to $60-80 \mathrm{~km} \mathrm{~s}^{-1}$ in about $10 \mathrm{Gyr}$. At the present level of observational accuracy the best we can say about the value of the heating index (defined as $\sigma(t)=\sigma_{0}(1+t / \tau)^{\alpha}$, in which $\sigma, t, \tau$, and $\alpha$ denote velocity dispersion, time, normalization constant, and heating index, respectively) is that it lies between $\alpha=0.3$ and $\alpha=0.5$ - the most recent studies favouring values closer to 0.3. It is thus not a very strong discriminator when comparing theoretical models or numerical simulations to the observational data. On the other hand, the ratio of vertical velocity dispersion to the radial velocity dispersion $\sigma_{z} / \sigma_{R}$ is better constrained observational quantity, being around $\sigma_{z} / \sigma_{R}=0.5 \pm 0.1$.

In the Paper I we simulated how the GMCs and the massive dark halo objects would heat up the stellar disk in the Solar neighbourhood. We verified the earlier results that due to the low number of GMCs in the Solar neighbourhood they can only heat the disk stars up to $\sim 40 \mathrm{~km} \mathrm{~s}^{-1}$ in $10 \mathrm{Gyr}$. We also found that $10^{6}$ Solar mass halo black holes are too inefficient in heating the stellar disk: the disk would be heated up to only $\sim 30 \mathrm{~km} \mathrm{~s}^{-1}$ in $10 \mathrm{Gyr}$ even if the whole dark halo were 
comprised of these black holes. Better results were achieved using $10^{7}$ Solar mass halo black holes. If half of the dark halo mass were made of them, the stellar disk would be heated up to $\sim 60 \mathrm{~km} \mathrm{~s}^{-1}$ in $10 \mathrm{Gyr}$, which would only be marginally consistent with observations.

Within observational uncertainties we could produce the right amount of heating by an appropriate combination of massive halo black holes $\left(M_{\mathrm{BH}}=1 \times 10^{7} M_{\odot}\right)$ and the known number density of GMCs in the Solar neighbourhood. Also the ratio $\sigma_{z} / \sigma_{R}$ and the heating index $\alpha$ fit the data within the observational error limits.

Because of the observational uncertainties the final conclusions about the role of putative massive halo objects (we will call these "massive halo objects" also black holes for brevity; they could be as well e.g. dark clusters as long as they are compact (of order of few parsecs)) in the heating process of the stellar disk have necessarily remained unclear. In this paper we have further investigated the implications of this heating process by asking how the massive halo objects would heat up the whole stellar disk, not just the Solar neighbourhood. We are aware of only one study in which the velocity dispersion of Galactic stellar disk has been observed at several different radii (see Lewis \& Freeman 1989). They observed the velocity dispersion in the plane of the old Galactic disk at eight different radii, ranging from about $1 \mathrm{kpc}$ to $17 \mathrm{kpc}$. We have compared our simulations to their observational data throughout this paper.

Wielen et al. (1992) have also discussed the radial dependency of the stellar velocity dispersion. They have estimated theoretically the diffusion process in the stellar disk due to massive halo black holes. Their prediction for the radial heating $\sigma_{R}$ as function of radius seems to be in accordance with the measurements by Lewis \& Freeman (1989) if black holes have mass $M_{\mathrm{BH}}=3 \times 10^{6} M_{\odot}$ and the dark halo density is proportional to $\rho_{\mathrm{BH}} \propto\left(a^{2}+R^{2}\right)^{-2}$ in which $a=3 \mathrm{kpc}$. Our results are similar to but not identical with theirs. This is not too surprising because our method is to simulate the physical processes of disk heating via GMCs and black holes, whereas Wielen et al. (1992) describe the heating analytically as a diffusion process in phase space.

\section{Numerical method}

\subsection{Local simulation method}

We use a simulation method in which all calculations are restricted to a small co-moving box within the Galactic disk. A typical computer run simulates a "patch" of the disk, being from $1 \times 1$ to $8 \times 8 \mathrm{kpc}$ square, and from 2 to $8 \mathrm{kpc}$ in vertical extent, containing of order of $10^{4}$ tracer stars in the disk as well as any perturbers (GMCs or BHs).

The orbits of stars in the patch are computed by integrating the linearized equations of motion (Hill 1878; Julian \& Toomre 1966):

$\ddot{x}-2 \Omega \dot{y}+\left(\kappa^{2}-4 \Omega^{2}\right) x=F_{x}$

$\ddot{y}+2 \Omega \dot{x}=F_{y}$

$\ddot{z}+v^{2} z=F_{z}$

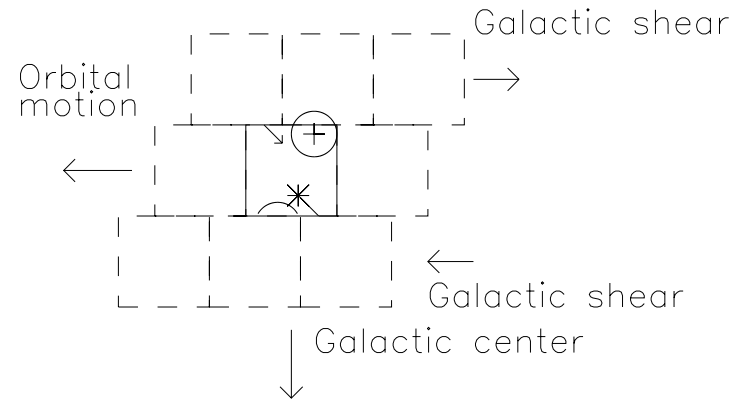

Fig. 1. The simulation box (solid line) and its surrounding virtual boxes (dashed lines) are illustrated. Gravitational forces on a given star (cross) are calculated from the gravitational perturbers whose nearest image lies within the distance $R_{g}$ (denoted by the circle). If a star (asterisk) leaves the simulation cell, its image will enter the cell in a way defined by the boundary conditions.

where $\Omega$ is the orbital frequency, and $\kappa$ and $v$ are the epicyclic frequency and the vertical frequency of motion, respectively. In the rotating Cartesian coordinate frame the $x$-axis points radially outward (away from the Galactic center), the $y$-axis in the direction of orbital motion, and the z-axis in the direction normal to the Galactic plane. The numerical integrator is the RK4 routine (Press et al. 1992). The tracer stars feel the Galactic potential and the forces due to perturbers (GMCs or black holes). In order to avoid numerical artifacts, the gravitational forces $\left(F_{x}, F_{y}, F_{z}\right)$ are calculated so that Newton's third law is satisfied, i.e. a perturber also feels the gravitational force from tracer stars.

\subsection{Boundary conditions}

Tracer stars and GMCs move on epicycles in the patch, and can potentially cross the patch's boundaries. Periodic boundary conditions are used to recover these stars/GMCs, which enter from the opposite boundary with a correction made for Galactic shear, as described in detail by Wisdom \& Tremaine (1988). The box in which the calculations are performed is thus effectively surrounded by virtual boxes which shear due to Galactic rotation, as illustrated in Fig. 1.

The BHs are on orbits characteristic of the dark halo, with high velocities relative to the disc stars. BHs move through the box on essentially straight line orbits. They obey the same periodic boundary conditions as the tracer stars in the radial $(x-)$ and azimuthal $(y-)$ direction, but not in the vertical $(z-)$ direction. The border in the $z$-direction is given special status, by setting it to be far enough from the disk midplane, in order that no forces need to be calculated across it. In practice one must take care that the box is large enough in the vertical direction, so that the stars never get close to the vertical boundary. When a halo black hole leaves the top or bottom of the box, it is removed from the simulation and a new one is randomly created from the parent velocity and space distribution. The number density of dark halo BHs entering and leaving the box is maintained at a constant level. 


\subsection{Numerical accuracy}

In typical unperturbed test runs the relative errors in vertical kinetic energy are of order of $10^{-7}$ - and much less in angular momentum. However, the error in the numerical integrations is dominated by the close encounters with the massive perturbers. The simplest way to monitor this error is to vary the integration step size in perturbed simulation runs and see if the evolution of the velocity dispersion is affected. The step size is considered short enough when further decrease does not alter the results. The optimal step size will depend both on the mass and size of the perturbing objects. Also a reasonable box size has to be found by experiment, so that the calculation of gravitational interaction is not cut off too shortly.

\section{Galactic model}

In a local simulation method the stars will feel the potential through the circular rotation rate $\Omega$, epicyclic frequency $\kappa$, and vertical frequency $v$. Values for these frequencies are wellknown only in the Solar neighbourhood - thus we need to use a model for the Galactic potential in order to establish frequencies at different radii from the Galactic center. We employ the model by Flynn et al. (1996).

The Galactic potential $\Phi$ is modelled by three components:

$\Phi=\Phi_{\mathrm{H}}+\Phi_{\mathrm{C}}+\Phi_{\mathrm{D}}$

in which $\Phi_{\mathrm{H}}, \Phi_{\mathrm{C}}$, and $\Phi_{\mathrm{D}}$ denote potentials of dark halo, central bulge, and disk, respectively. The potentials of dark halo and central bulge are assumed to be spherical. A logarithmic potential is used for the halo:

$\Phi_{\mathrm{H}}=\frac{1}{2} V_{\mathrm{H}}^{2} \ln \left(r^{2}+r_{0}^{2}\right)$,

where $r$ is the 3D Galactocentric radius, $r_{0}$ the core radius, and $V_{\mathrm{H}}$ is the circular velocity at large $r$. Two Plummer potentials have been used for the central bulge:

$\Phi_{\mathrm{C}}=-\frac{G M_{\mathrm{C}_{1}}}{\sqrt{r^{2}+r_{\mathrm{C}_{1}}^{2}}}-\frac{G M_{\mathrm{C}_{2}}}{\sqrt{r^{2}+r_{\mathrm{C}_{2}}^{2}}}$,

where $r_{\mathrm{C}_{\mathrm{i}}}, i=1,2$, is the bulge core radius, $M_{\mathrm{C}_{\mathrm{i}}}, i=1,2$, is the mass of the bulge, and gravitational constant is denoted by $G$. The disk potential is modelled by a combination of three Miyamoto-Nagai potentials:

$\Phi_{\mathrm{D}}=\Phi_{\mathrm{D}_{1}}+\Phi_{\mathrm{D}_{2}}+\Phi_{\mathrm{D}_{3}}$,

where

$\Phi_{\mathrm{D}_{n}}=\frac{-G M_{\mathrm{D}_{n}}}{\sqrt{R^{2}+\left[a_{n}+\sqrt{z^{2}+b^{2}}\right]^{2}}}, \quad n=1,2,3$.

The disk scale height is same for all the components $n=1,2,3$ and is denoted by $b$, but the disk scale lengths $a_{n}$ are different. Now $R$ is cylindrical radius and $z$ the vertical coordinate $\left(r^{2}=\right.$ $R^{2}+z^{2}$ ). The parameters for the potentials are shown in Table 1 . Even though the mass $M_{\mathrm{D}_{2}}$ is set to a negative value the density resulting from the total disk potential is positive everywhere.
Table 1. Parameters for the Galactic potential.

\begin{tabular}{lll}
\hline \hline Component & \multicolumn{2}{l}{ Parameter Value } \\
\hline Dark halo & $r_{\circ}$ & $8.5 \mathrm{kpc}$ \\
& $V_{\mathrm{H}}$ & $220 \mathrm{~km} \mathrm{~s}^{-1}$ \\
Central bulge & $r_{\mathrm{C}_{1}}$ & $2.7 \mathrm{kpc}$ \\
& $M_{\mathrm{C}_{1}}$ & $3.0 \times 10^{9} M_{\odot}$ \\
& $r_{\mathrm{C}_{2}}$ & $0.42 \mathrm{kpc}$ \\
& $M_{\mathrm{C}_{2}}$ & $1.6 \times 10^{10} M_{\odot}$ \\
Disk & $b$ & $0.3 \mathrm{kpc}$ \\
& $M_{\mathrm{D}_{1}}$ & $6.6 \times 10^{10} M_{\odot}$ \\
& $a_{1}$ & $5.81 \mathrm{kpc}$ \\
& $M_{\mathrm{D}_{2}}$ & $-2.9 \times 10^{10} M_{\odot}$ \\
& $a_{2}$ & $17.43 \mathrm{kpc}$ \\
& $M_{\mathrm{D}_{3}}$ & $3.3 \times 10^{9} M_{\odot}$ \\
& $a_{3}$ & $34.86 \mathrm{kpc}$ \\
\hline
\end{tabular}

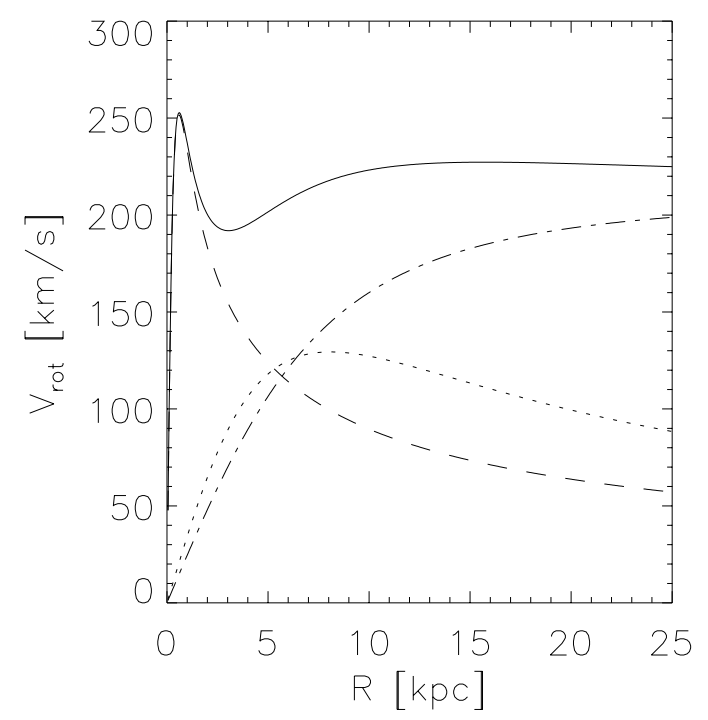

Fig. 2. The rotation curve of the model by Flynn et al. (1996). The solid line is the total rotation curve. The contributions from the dark halo, the central bulge, and the disk are indicated by dash-dotted, dashed, and dotted lines, respectively.

Flynn et al. (1996) chose the parameters to be consistent with the dynamical mass measurements of the disk surface density in the Solar neighbourhood, and to fit well the Galactic rotation curve. Most importantly, the model had to reproduce a realistic disk scale length.

The rotation curve of the model is shown in Fig. 2. The local surface density for the Solar radius $\left(R_{\odot}=8 \mathrm{kpc}\right)$ is $\Sigma_{\mathrm{D}}=52 M_{\odot} / \mathrm{pc}^{2}$ which is consistent with recent measurements (Kuijken \& Gilmore 1989; Flynn \& Fuchs 1994; Holmberg $\&$ Flynn 2000). The model for the potential produces a disk surface density that is exponential and has a scale length of $4.1 \mathrm{kpc}$. The disk also has a uniform scale height of order 260 pc.

Values for $\Omega, \kappa$, and $v$ derived from the model are plotted in Fig. 3. Numerical values used in the local-patch simulations are shown in Table 2 at different radii. The density $\rho_{\mathrm{D}}$ at $z=0$ and the surface density $\Sigma$ of the disk derived from the Galactic 


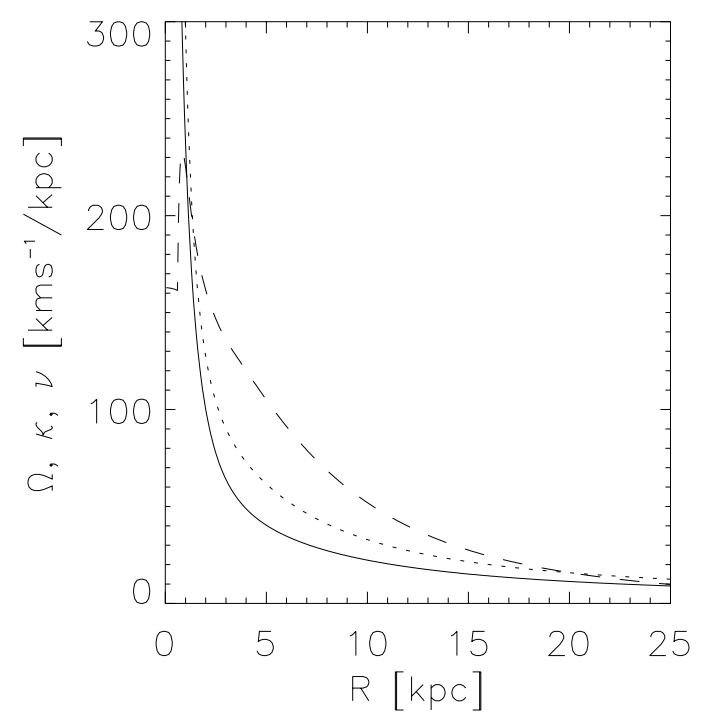

Fig. 3. The orbital $(\Omega)$, epicyclic $(\kappa)$, and vertical $(v)$ frequencies derived from the model by Flynn et al. (1996) are plotted by solid, dotted, and dashed lines, respectively.

Table 2. Orbital, epicyclic, and vertical frequencies derived from the model.

\begin{tabular}{|c|c|c|c|}
\hline$R$ & $\Omega$ & $\kappa$ & $v$ \\
\hline [kpc] & {$\left[\mathrm{km} \mathrm{s}^{-1} / \mathrm{kpc}\right]$} & {$\left[\mathrm{km} \mathrm{s}^{-1} / \mathrm{kpc}\right]$} & {$\left[\mathrm{km} \mathrm{s}^{-1} / \mathrm{kpc}\right]$} \\
\hline 4 & 48.79 & 72.89 & 120.07 \\
\hline 6 & 34.67 & 53.11 & 91.24 \\
\hline 8 & 27.21 & 41.00 & 68.60 \\
\hline 12 & 18.83 & 27.24 & 39.89 \\
\hline 16 & 14.20 & 20.06 & 24.47 \\
\hline
\end{tabular}

model are shown in Table 3. Also the corresponding values of the vertical component of the velocity dispersion $\sigma_{z}$ are shown for the vertical scale height $z_{0}=260 \mathrm{pc}$.

The values for $\Omega, \kappa$, and $v$ are derived from the analytic potential $\Phi$, which represents the smooth, stationary potential of the Galaxy. The potential thus lacks the effects due to coarsegraininess of the Galactic mass distribution, which is actually responsible for the disk heating. In our simulations the coarsegraininess of the potential is introduced in the form of perturbers in the local patch. The patch only ever includes at most a handful of perturbers, and their explicit effect on $\Omega, \kappa$, and $v$ is negligible; these quantities can be computed sufficiently accurately for our purposes from the background potential represented by $\Phi$.

\section{Observational data}

Throughout the paper we use the observational data by Lewis \& Freeman (1989) as a comparison to our simulations. Lewis and Freeman observed about 600 old disk K giants with broadband photometry and medium-resolution spectroscopy. All these $\mathrm{K}$ giants were located at Galactic low-absorption windows in the plane of the disk. They observed among other things radial $\sigma_{R}$ and tangential $\sigma_{\phi}$ components of the velocity
Table 3. Disk densities at $z=0$ and corresponding vertical velocity dispersion at $z=0$.

\begin{tabular}{llll}
\hline \hline$R$ & $\rho_{D}(0)$ & $\Sigma$ & $\sigma_{z}$ \\
{$[\mathrm{kpc}]$} & {$\left[M_{\odot} / \mathrm{pc}^{3}\right]$} & {$\left[M_{\odot} / \mathrm{pc}^{2}\right]$} & {$\left[\mathrm{km} \mathrm{s}^{-1}\right]$} \\
\hline 4 & 0.267 & 158.5 & 23.6 \\
6 & 0.154 & 91.9 & 18.0 \\
8 & 0.087 & 52.0 & 13.5 \\
12 & 0.029 & 17.6 & 7.9 \\
16 & 0.011 & 6.6 & 4.8 \\
\hline
\end{tabular}

dispersion at several radii, ranging from $R=0.6 \mathrm{kpc}$ to $R=$ $17.7 \mathrm{kpc}$; these are the data which primarily constrain our simulations.

They found that the radial and tangential velocity dispersions in the disk fall exponentially with radius $R$. They fit their velocity dispersion data to

$\sigma=\sigma_{0} \mathrm{e}^{-R / 2 h}$,

in which $h$ denotes disk scale length. For the radial velocity dispersion they get $h_{R}=4370 \pm 320 \mathrm{pc}$. For the tangential velocity dispersion their value is $h_{\phi}=3360 \pm 620 \mathrm{pc}$. The difference in the radial and tangential determinations of the scale length they explain to be result of the fact that the rotation curve is not precisely flat over the observed region of the Galaxy.

The determination of the tangential velocity dispersion from the observational data is more complicated than the radial velocity dispersion. The radial velocity dispersion is calculated in the fields in which the azimuthal contribution to the velocity dispersion is insignificant. On the other hand, when calculating the tangential velocity dispersion the contribution from the radial velocities has to be taken into account. This is done by assigning to each star in the sample a radial random velocity by the Monte Carlo method. The earlier determined mean radial velocities and velocity dispersions are used in defining the distribution from which the values are drawn. Because the determination of the radial velocity dispersion is thus a considerably more straight forward procedure we put more emphasis on it when comparing our results to the observations.

The determination of the disk scale length from the observational data is based on two assumptions: Firstly, it is assumed that the radial variation of the vertical velocity dispersion is proportional to $\sigma_{z}^{2} \propto \mathrm{e}^{-R / h}$ due to hydrostatic equilibrium. Secondly, one has to assume that the form of the velocity ellipsoid is constant throughout the disk, i.e. $\sigma_{z} / \sigma_{R} \approx$ constant. After these steps we can use Eq. (7) to calculate the radial scale length.

However, there are some difficulties when comparing the scale lengths determined from the simulations to the observational one. Our simulations indicate that the ratio $\sigma_{z} / \sigma_{R}$ is not necessarily constant. Thus the assumption used when the disk scale length is derived from the observations is somewhat problematic. This should be kept in mind when the simulations are compared to the observations.

In Paper I we discussed the observational data on the stellar heating in the Solar neighbourhood. The data indicated that 
the oldest stellar populations had total velocity dispersion of order of $\sigma_{\text {tot }} \sim 70 \mathrm{~km} \mathrm{~s}^{-1}$. Lewis \& Freeman, on the other hand, adopted a smaller value for the velocity dispersion in the Solar neighbourhood. For the radial component their best fit to the data gives $\sigma_{R} \simeq 42 \mathrm{~km} \mathrm{~s}^{-1}$, and $\sigma_{\phi} \simeq 33 \mathrm{~km} \mathrm{~s}^{-1}$. Assuming $\sigma_{z} / \sigma_{R}=0.6$ their total velocity dispersion will be $\sigma_{\text {tot }} \sim 60 \mathrm{~km} \mathrm{~s}^{-1}$. When comparing the simulation results to the observational data in the paper I we concluded that halo black holes of mass $M_{\mathrm{BH}}=1 \times 10^{7} M_{\odot}$ together with GMCs are needed to explain the observed amount of heating. If compared to the Lewis \& Freeman data for the values in the Solar neighbourhood we could have reproduced the observed heating with less or lower mass halo black holes.

\section{Heating of the disk due to massive halo objects}

Originally Lacey \& Ostriker (1985) used massive halo objects to explain the stellar heating in the Galactic disk. This halo black hole scenario has also some difficulties which we will discuss.

One of the strongest arguments against the halo black hole scenario is the presence of globular clusters. For example, Moore (1993) has estimated the upper limit for the mass of halo objects to be $\sim 10^{3} M_{\odot}$, otherwise several of the low-mass globular clusters would have heated into disruption in close encounters with massive halo objects. He used numerical simulations to investigate how much kinetic energy an encountering black hole would transmit into a globular cluster. Monte Carlo simulations were used to estimate how much time is needed for halo black holes to disrupt a globular cluster in subsequent encounters, i.e. how much time is needed for the globular cluster to gain kinetic energy of order its binding energy. The upper limit of a halo black hole mass is then estimated by requiring the smallest existing globular cluster to survive the subsequent halo black hole encounters for $7 \mathrm{Gyr}$ (half of the typical age of the halo globular cluster).

Even though the above mentioned study presents a strong case against the plausible halo black hole population, we can argue that the present day globular cluster population is only a remnant of a once larger population. In fact, the globular cluster system in the Galaxy does not follow pure a powerlaw near the center, but the distribution is centrally flattened (Meylan \& Heggie 1997). In several other galaxies the globular cluster system is also found to be much less centrally concentrated than the light, indicating that the globular cluster systems have evolved during their life times (see e.g. McLaughlin et al. 1994). In addition to a halo black hole population also tidal forces due to e.g. a supermassive black hole at the center of a galaxy or a Galactic bulge can affect the globular cluster distribution (Charlton \& Laguna 1995).

Concerning the internal dynamics of globular clusters, it is now recognized that there is a substantial fraction of binaries in the globular clusters (e.g. Rubinstein and Bailyn (1997) derived the fraction to be $15 \%-38 \%$ for the core of NGC 6752). Even though the role of binaries in the evolution of globular clusters is still somewhat poorly understood, we are sure that binaries play a crucial role. Binaries can e.g. prevent core-collapse because the binary population can absorb, store, and emit kinetic energy within the system. Moore's simulations (1993) lack the binary population which might have affected the stability of his globular clusters against external perturbations. In this case he would have yielded a too low value for the upper limit of the sustainable halo black hole mass.

One argument against putative halo black holes is the fact that some galaxies have remarkable thin disks, or that if dark halos of dwarf spiral galaxies were dominated by similar black holes their disks would be easily destroyed (Friese et al. 1995). A way around this is to question the assumption made in those studies that the BH mass is the same in all dark halos.

BHs passing through the disk might also be expected to accrete and reveal themselves as high proper motion X-ray sources, and no such objects have been seen. It seems worth noting though that the central black hole in the Milky Way demonstrates that the accretion rate and emergent flux from a black hole of about the desired mass $\left(2.6 \times 10^{6} M_{\odot}\right)$ is far from well understood (Genzel 1998); the central black hole in the Milky Way is remarkably quiet in X-rays.

In order to avoid the difficulties with the halo black hole scenario it was later proposed that the stellar heating would be due to massive dark clusters orbiting inside the halo (Carr \& Lacey 1987). The dark clusters should be quite compact for this model to work: their core radius should be at maximum $r_{\mathrm{c}} \sim 1 \mathrm{pc}$ and the components should have masses less than $\sim 10 M_{\odot}$.

The latest generation of cosmological simulations of galaxy formation do indeed show quite clumpy dark halos around Milky Way type spirals (Moore et al. 1999). The amount, size and distribution of these clumps is still very uncertain, mainly due to numerical resolution issues, but if they can survive in the inner regions of dark halos they may well be able to heat disks in a manner consistent with observations.

Even though there are arguments against the possible existence of massive compact halo objects the debate is still unfinished. With this in mind we have taken a look at the consequencies of such putative halo black hole population on the disk heating. In Paper I the strength of our conclusions were weakened because of ambiguous observational results. Now we have retested the halo black hole hypothesis by comparing to new observational data.

\subsection{Initial values}

For the massive halo objects we have used isotropic velocity dispersions of $\sigma_{x, y, z}=135 \mathrm{~km} \mathrm{~s}^{-1}$ at all radii. But what initial velocity dispersion should be used for the stellar population in the disk? In a self-consistent disk model the radial velocity dispersion should be proportional to the surface density, i.e. $\sigma_{R}^{2} \propto \mathrm{e}^{-R / h}$. In an isothermal model also the vertical velocity dispersion is related to the disk surface density, i.e. $\sigma_{z}=\pi G \Sigma(R) z_{0}$ (see Table 3 ). Now the disk has constant scale height $z_{0}$ and the ratio of the vertical velocity dispersion to the radial velocity dispersion is constant throughout the disk.

However, observational evidence reveals that the molecular hydrogen does not have the velocity dispersion required by the self-consistent model. It is well-known that the atomic 
and molecular hydrogen is concentrated in a thin layer within the Galactic disk. For example, Sanders et al. (1984) find that the vertical thickness of the molecular gas layer increases only from 40 to $75 \mathrm{pc}$ between $R=3$ and $9.5 \mathrm{kpc}$. The onedimensional velocity dispersion of molecular clouds has been measured to be about $5 \mathrm{~km} \mathrm{~s}^{-1}$ (Sanders et al. 1984). Outside the Solar circle the molecular hydrogen forms a somewhat thicker layer, from $77 \mathrm{pc}$ at $R=9 \mathrm{kpc}$ to $188 \mathrm{pc}$ at $R=18 \mathrm{kpc}$, having maximum thickness of $257 \mathrm{pc}$ at $R=15 \mathrm{kpc}$ according to Wouterloot et al. (1990).

The vertical velocity dispersion of molecular clouds is thus rather constant and decoupled from the disk potential. Narayan \& Jog (2002) have been able to reproduce analytically the observed distribution of atomic and molecular gas layers: remarkable small vertical scale height with some flaring. They argue that the gravitational potential of atomic and molecular hydrogen has to be taken into account together with the stellar disk potential. The response of each component to the joint potential is then different because of their different velocity dispersions.

Because stars must initially have the same velocity dispersion as the molecular clouds they have been born in, our stellar population has thus constant initial vertical velocity dispersion with respect to Galactocentric radius. In practice, we adopt an initial radial velocity dispersion of $\sigma_{R}=12 \mathrm{~km} \mathrm{~s}^{-1}$ and vertical velocity dispersion of $\sigma_{z}=5 \mathrm{~km} \mathrm{~s}^{-1}$ to the stellar population. The azimuthal velocity dispersion then follows from $\sigma_{R}, \Omega$, and $\kappa$.

\subsection{Halo of $10^{6} M_{\odot} B H s$}

The dark halo density is normalized based on the mass density of the dark halo in the Solar neighbourhood: it has been established to be $\rho_{\mathrm{H}} \sim 0.01 M_{\odot} \mathrm{pc}^{-3}$ (Holmberg \& Flynn 2000). We have used a density $\rho_{\mathrm{H}}=0.008 M_{\odot} \mathrm{pc}^{-3}$ at the Solar radius for our dark halo consisting of black holes of mass $M_{\mathrm{BH}}=1 \times 10^{6} M_{\odot}$. Thus about $80 \%$ of the dark halo is made of these objects. The dark halo density, i.e. number density of $\mathrm{BHs}$, is then scaled as $\rho_{\mathrm{H}} \propto r^{-2}$ for the appropriate Galactic radius.

The radial component of the velocity dispersion is plotted as a function of Galactocentric radius in Fig. 4. The velocity dispersion from simulations (plotted with diamonds) is shown after evolution of 11.29 billion years which corresponds to 50 orbital periods at the Solar radius $\left(R_{\odot}=8 \mathrm{kpc}\right)$. The least-square-fit to the simulated values is shown by the solid line. For comparison, the Lewis and Freeman (1989) data are plotted as squares, and their own fit to their data is shown with a dashed line. Also the estimated errors they give are shown.

The observed velocity dispersion is decreasing more strongly outward than the simulated velocity dispersion. The difference in the slopes of the curves is significant: the simulation gives $h_{R}=6100 \mathrm{pc}$ for the scale length. Furthemore, the heating induced by halo BHs (of mass $10^{6} M_{\odot}$ ) is clearly insufficient especially in the inner parts of the Galactic disk.

The same tendency is also noticed when the observed and simulated tangential components of the velocity dispersion are compared in Fig. 5: the observed velocity dispersion has a

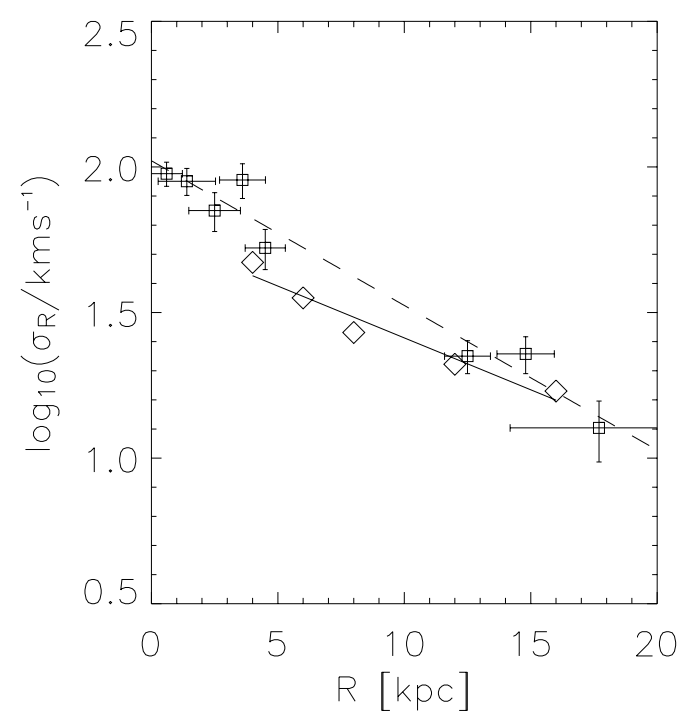

Fig. 4. Disk heating resulting from $10^{6} M_{\odot}$ mass halo black holes is compared with the observations as a function of Galactocentric radius $R$. Diamonds denote simulation results and a solid line is the RMS-fit to them. The fit to the observational data (squares, shown with error bars) is shown by a dashed line.

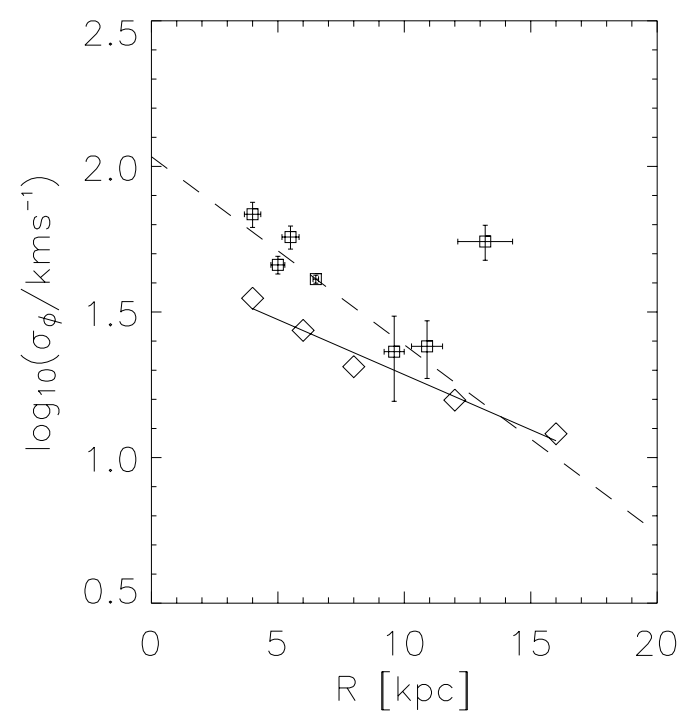

Fig. 5. The azimuthal velocity dispersions resulting from heating by $10^{6} M_{\odot}$ mass halo black holes, compared with the observations. Diamonds denote simulation results and the solid line is the rms-fit to them. The fit to the observational data (squares, shown with error bars) has been drawn with dashed line. Lewis \& Freeman have omitted the data point at $R=13.2 \mathrm{kpc}$ from their fit.

steeper slope than the simulations show as a function of radius. We obtain $h_{\phi}=5740$ pc for the tangential scale length. Interestingly Lewis and Freeman (1989) get about as large values for the tangential velocity dispersion as for the radial velocity dispersion in the inner parts of the Galaxy. This might be due to some non-axisymmetric features in the Galactic potential. In our simulations the tangential velocity dispersion is consistently smaller than the radial velocity dispersion, as should be the case in the axisymmetric potential. 


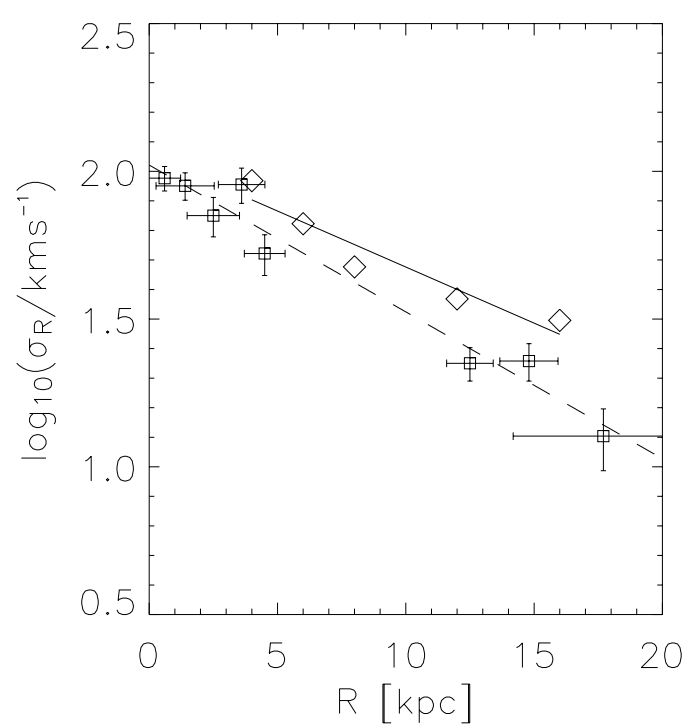

Fig. 6. The radial heating resulting from $10^{7} M_{\odot}$ mass halo black holes is compared with the observations. Diamonds denote simulation results and the solid line is the rms-fit to them. The fit to the observational data (shown with error bars) has been drawn with a dashed line.

\subsection{Halo of $10^{7} M_{\odot} B H s$}

For a dark halo consisting of BHs of mass $M_{\mathrm{BH}}=1 \times 10^{7} M_{\odot}$ we have used a dark matter density $\rho_{\mathrm{H}}=0.005 M_{\odot} \mathrm{pc}^{-3}$ in the Solar neighbourhood. This density corresponds to a dark halo which has $50 \%$ of its mass in the form of massive black holes. Note that for the BHs of mass $M_{\mathrm{BH}}=1 \times 10^{6} M_{\odot}$ we used a different normalization. These normalizations are as were used in Paper I.

The comparison between simulated and Lewis \& Freeman (1989) values for the radial component of velocity dispersion is shown in Fig. 6. In this scenario the BHs heat up the disk more than the observational evidence indicates, especially in the outer parts of disk. The slope of the radial velocity dispersion fit as function of radius is slightly steeper than in the previous case - now the radial scale length is $h_{\mathrm{R}}=5720 \mathrm{pc}$. The observations indicate a still steeper slope.

The tangential component of the velocity dispersion, on the other hand, falls within the observational limits in the inner parts of the Galaxy: the scale length $h_{\phi}=5320 \mathrm{pc}$ is still larger than measured by Lewis \& Freeman (1989). However, the observed tangential velocity dispersion (Lewis \& Freeman 1989) is more model dependent than the radial component, so more weight should be put on the radial velocity dispersion.

\subsection{Vertical velocity dispersion}

The ratio of vertical velocity dispersion to the radial velocity dispersion is plotted in Figure 8 for both the cases (halo containing $M_{\mathrm{BH}}=1 \times 10^{6} M_{\odot}$ BHs and halo containing $\left.M_{\mathrm{BH}}=1 \times 10^{7} M_{\odot} \mathrm{BHs}\right)$. Diamonds with the solid line fit denote the former case and triangles with the dashed line fit refer to the latter case. Lewis and Freeman (1989) did not observe the vertical velocity dispersion.

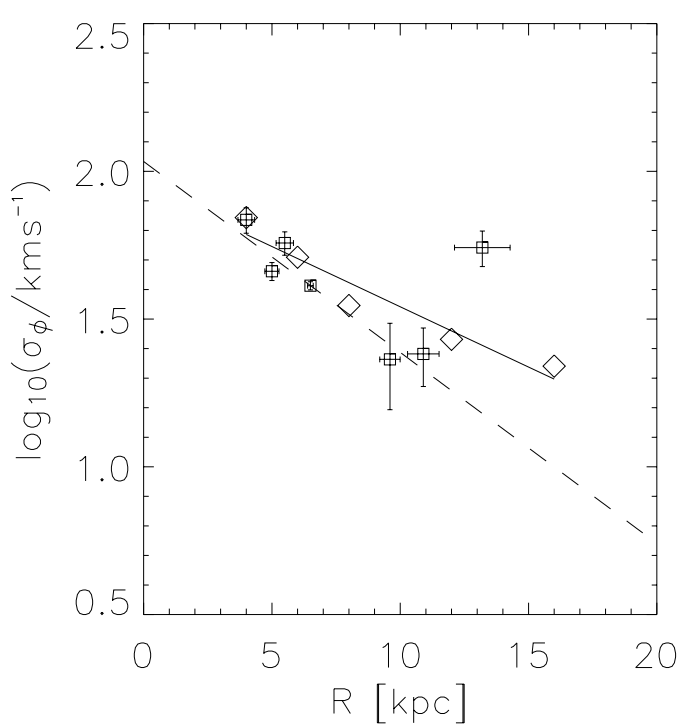

Fig. 7. The azimuthal heating resulting from $10^{7} M_{\odot}$ mass halo black holes is compared with the observations. Diamonds denote simulation results and a solid line is the rms-fit to them. The fit to the observational data (shown with error bars) has been drawn with dashed line.The observational data point at $r=13.2 \mathrm{kpc}$ has been omitted from the fit.

The heavier BHs produce a higher $\sigma_{z} / \sigma_{R}$ ratio as expected. We demonstrated already in the Paper I that more massive perturbers do not just give rise to a higher velocity dispersion but also give rise to a higher $\sigma_{z} / \sigma_{R}$ ratio. The slope of the $\sigma_{z} / \sigma_{R}$ curves is also decreasing outward with radius. The decrease is significant even if not dramatic. It is due to the fact that as the density of the halo BHs is proportional to $\rho_{\mathrm{H}} \propto r^{-2}$ and thus the $\mathrm{BH}$ number density decreases outward. The $\sigma_{z} / \sigma_{R}$ ratio was also found to depend on the number density of the perturbers (Paper I), i.e. higher density increases the $\sigma_{z} / \sigma_{R}$ ratio. The $\sigma_{z} / \sigma_{R}$ ratio must thus decrease outward. The scale length of the disk derived from the vertical velocity dispersion is $h_{z}=5150 \mathrm{pc}$ and $h_{z}=4920 \mathrm{pc}$ for the $M_{\mathrm{BH}}=1 \times 10^{6} M_{\odot}$ and $M_{\mathrm{BH}}=1 \times 10^{7} M_{\odot}$ halos, respectively. The decrease in the $\sigma_{z} / \sigma_{R}$ ratio as a function of radius also explains the smaller scale lengths when they are calculated from the vertical velocity dispersion.

\section{Heating of the disk due to GMCs}

Most of the molecular gas in the Galaxy is concentrated at the Galactic center and in the ring at a Galactocentric distance extending from about 3 to about $6.5 \mathrm{kpc}$ (Solomon et al. 1979; Sanders et al. 1984; note that we have scaled the Solar distance from $R_{\odot}=10 \mathrm{kpc}$ to $\left.R_{\odot}=8 \mathrm{kpc}\right)$. The Sun is located at the outer edge of the ring of the molecular gas, where the radial distribution of molecular gas is falling steeply. Outside the Solar circle the density of the molecular gas is negligible. Table 4 shows the radial density distribution of molecular hydrogen according to Scoville \& Sanders (1986).

Molecular gas lies in a very thin layer with a onedimensional velocity dispersion of only about $5 \mathrm{~km} \mathrm{~s}^{-1}$. Another property of the molecular gas is that most of the mass 


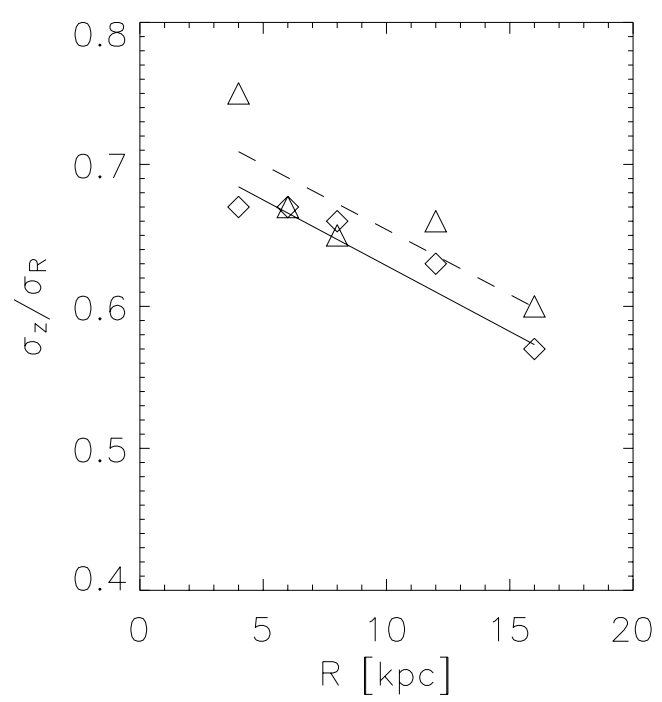

Fig. 8. The simulated ratios of vertical velocity dispersion to the radial velocity dispersion are plotted in tha cases of $10^{6} M_{\odot}$ and $10^{7} M_{\odot}$ mass halo black holes. Diamonds denote simulations of halo black holes with mass $10^{6} M_{\odot}$ and triangles denote black holes of mass $10^{7} M_{\odot}$. The least-square-fit drawn with the solid line refers to the former case and the dashed line refers to the latter case.

Table 4. Radial density distribution of molecular hydrogen $\Sigma_{\mathrm{H}_{2}}$ from Scoville and Sanders (1986). The Solar radius is scaled from $10 \mathrm{kpc}$ to $8 \mathrm{kpc}$. $\Sigma_{\mathrm{GMC}}$ denote the surface density used for the GMCs in the simulations.

\begin{tabular}{|c|c|c|}
\hline $\begin{array}{l}R \\
{[\mathrm{kpc}]}\end{array}$ & $\begin{array}{l}\Sigma_{\mathrm{H}_{2}} \\
{\left[M_{\odot} / \mathrm{pc}^{2}\right]}\end{array}$ & $\begin{array}{l}\Sigma_{\mathrm{GMC}} \\
{\left[M_{\odot} / \mathrm{pc}^{2}\right]}\end{array}$ \\
\hline 1.6 & 5.0 & - \\
\hline 2.4 & 6.0 & - \\
\hline 3.2 & 9.0 & - \\
\hline 4.0 & 16.0 & 12.0 \\
\hline 4.8 & 23.0 & - \\
\hline 5.6 & 17.0 & - \\
\hline 6.0 & - & 12.0 \\
\hline 6.4 & 12.0 & - \\
\hline 7.2 & 7.0 & - \\
\hline 8.0 & 5.0 & 4.0 \\
\hline 8.8 & 1.0 & - \\
\hline 9.6 & 1.0 & - \\
\hline
\end{tabular}

of the molecular hydrogen is concentrated in the giant molecular clouds. Generally molecular gas clouds with masses from $10^{5} M_{\odot}$ to $4 \times 10^{6} M_{\odot}$ and sizes from 20 to $100 \mathrm{pc}$ are termed giant molecular clouds (Scoville \& Sanders 1986). In our simulations we have modelled the GMCs to be homogeneous spheres with mass $M_{\mathrm{GMC}}=1 \times 10^{6} M_{\odot}$ and diameter of $100 \mathrm{pc}$. We have taken $80 \%$ of the actual observed molecular gas density for the value we have used in the simulations, because some fraction of mass is in the smaller gas clouds and can have no effect on the evolution of the stellar velocity dispersion. The values used in the simulations are shown in Table 4. Outside the Solar radius the number of GMCs has been set to zero in the simulations.

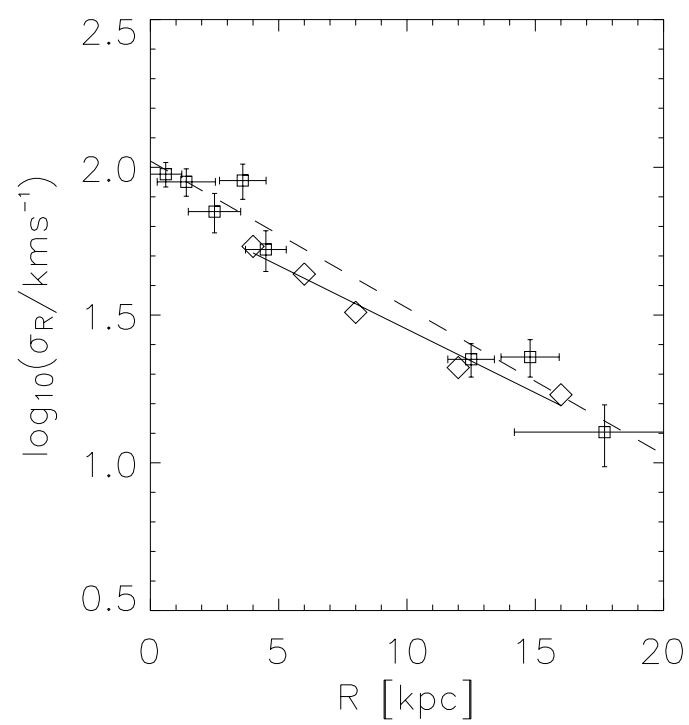

Fig. 9. The radial heating resulting from $10^{6} M_{\odot}$ mass halo black holes and GMCs is compared with the observations. Diamonds denote simulation results and a solid line is the least-square-fit to them. The dashed line is the fit to the observational data (squares and error bars).

Giant molecular clouds do heat up the stellar disk, but this heating has been found to be inconsistent with the observations. First of all, there just is not enough GMCs in the Solar neighbourhood to explain the observed values of the velocity dispersion of the older stellar populations. Also the heating due to GMCs will produce a heating index that is too small when compared to the observations (see Paper I). However, the heating due to GMCs is significant enough so that it contributes to the overall picture of stellar heating in the Galactic disk, especially when there is a strong radial gradient in the molecular gas distribution.

\subsection{Halo of $10^{6} M_{\odot} B H s$}

The inclusion of GMCs corrects the heating effect towards the observed values. The radial velocity dispersion is now almost within the observed limits, as seen in Fig. 9. Also the $\sigma_{R}-R$ curve now has a steeper slope than when only the halo BHs were acting as perturbers. The slope of the fit correponds now to the scale length $h_{R}=5050 \mathrm{pc}$ which is only $\sim 700 \mathrm{pc}$ larger than Lewis \& Freeman's value. They give 320 pc as their formal error limit, so strictly speaking our value is only consistent at about $2-\sigma$ level. The simulated scale length $h_{\phi}=4780 \mathrm{pc}$ determined from the tangential velocity dispersion is again further off from their value (see Fig. 10) by slightly more than 2- $\sigma$ level.

\subsection{Halo of $10^{7} M_{\odot} B H s$}

If we increase the mass of BHs to $10^{7} M_{\odot}$ the total heating effect will naturally increase and the heating due to GMCs will become relatively less effective. It is now seen to exceed the observed amount of heating in Fig. 11. The radial scale length will thus tend to increase when compared to simulations with BHs of mass $10^{6} M_{\odot}$ (and GMCs). We have measured the scale 


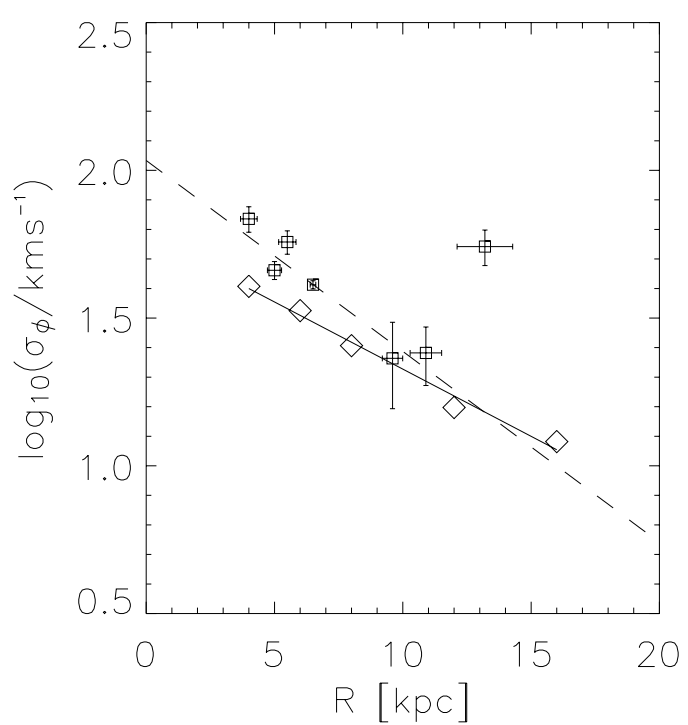

Fig. 10. The azimuthal heating resulting from $10^{6} M_{\odot}$ mass halo black holes and GMCs is compared with the observations. Diamonds denote simulation results and a solid line is the least-square-fit to them. The dashed line is the fit to the observational data (squares and error bars). The single deviant data point has been ignored in the fitting, following Lewis and Freeman.

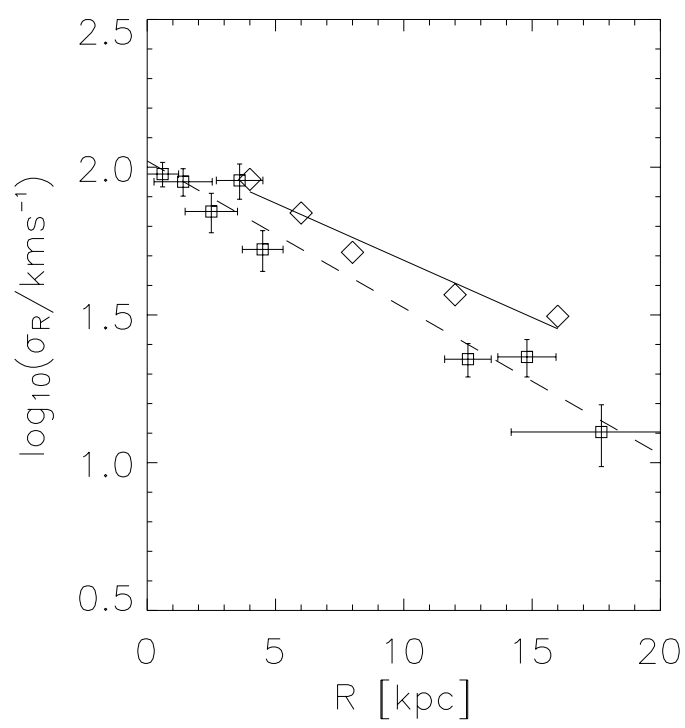

Fig. 11. The radial heating resulting from $10^{7} M_{\odot}$ mass halo black holes and GMCs is compared with the observations. Diamonds denote simulation results and a solid line is the least-square-fit to them. The dashed line shows the fit to the observational data (squares with error bars).

length to be $h_{R}=5620 \mathrm{pc}$, which is only slightly less than in the simulations with only dark halo perturbers of similar mass.

The same is also true with the azimuthal scale length as seen in Fig. 12. We obtain the scale length $h_{\phi}=5270$ pc. It is only slightly less than the value obtained from the simulation without GMCs, of 5320 pc.

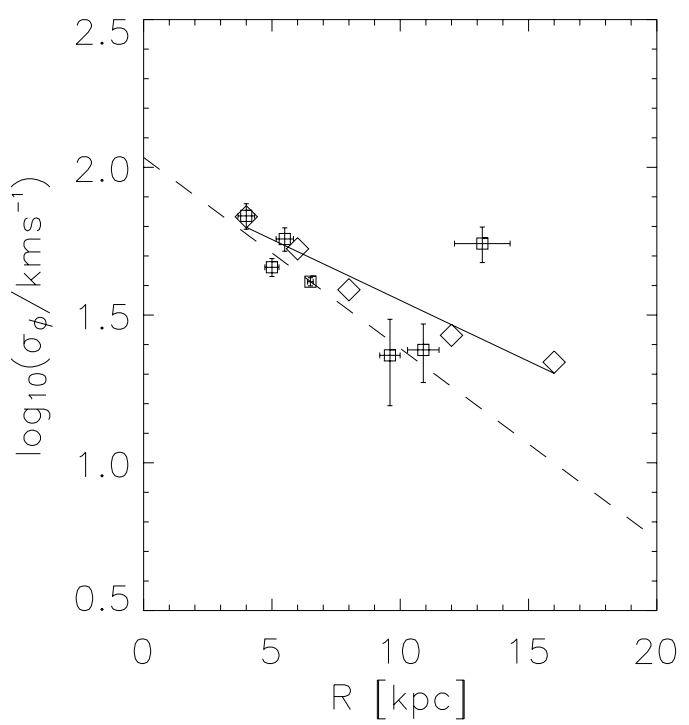

Fig. 12. The azimuthal heating resulting from $10^{7} M_{\odot}$ mass halo black holes and GMCs is compared with the observations. Diamonds denote simulation results and a solid line is the least-square-fit to them. The dashed line shows the fit to the observational data (squares with error bars). The observational data point at $r=13.2 \mathrm{kpc}$ has been omitted from the fit by Lewis \& Freeman.

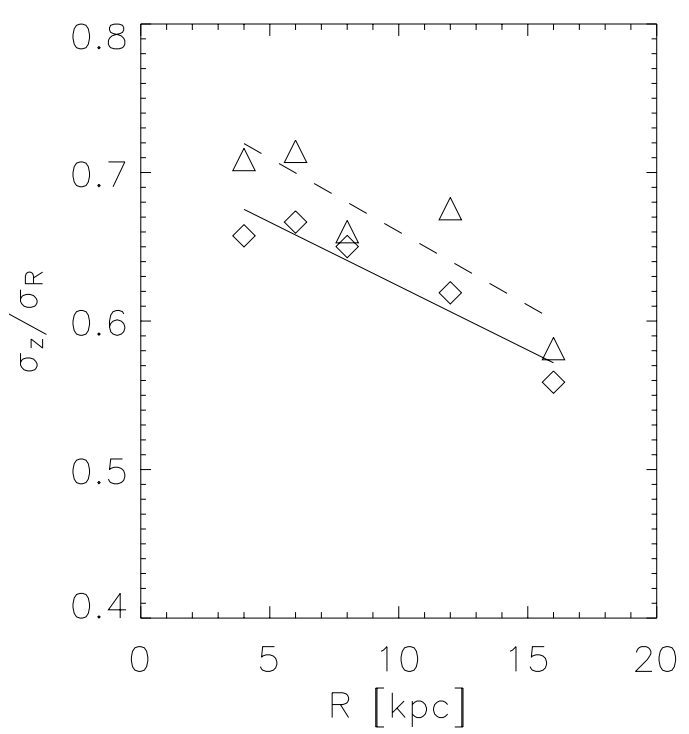

Fig. 13. The simulated ratios of vertical velocity dispersion to the radial velocity dispersion are plotted in the cases of GMCs together with $10^{6} M_{\odot}$ and $10^{7} M_{\odot}$ mass halo black holes. Diamonds denote simulations of halo black holes with mass $10^{6} M_{\odot}$ and triangles denote the case with black holes of mass $10^{7} M_{\odot}$. The solid line refers to the least-square-fit in the former case and the dashed line refers to the latter case.

\subsection{Vertical velocity dispersion}

The GMC distribution does not radically alter the $\sigma_{z} / \sigma_{R}$-ratio, as plotted in Fig. 13, compared to the case having only halo perturbers (Fig. 8). However, further analysis shows that the radial scale length calculated from the vertical velocity dispersion is affected. The scale length is now $h_{z}=4420 \mathrm{pc}$ for the $10^{6} M_{\odot}$ BHs. It has shortened about $600 \mathrm{pc}$ from what is was in the case without GMCs. This value is actually well within 
the error limits with the scale length derived from the radial velocity dispersion by Lewis \& Freeman.

In the simulation runs of $10^{7} M_{\odot}$ halo BHs the GMC distribution also act to shorten the scale length, up to $h_{z}=4800 \mathrm{pc}$, i.e. it has been shortened now only about $100 \mathrm{pc}$. This value is within $1.5-\sigma$ of the Lewis \& Freeman value.

\section{Conclusions}

In Paper I we investigated stellar heating in the Solar neighbourhood. We found that within observational limits the observed stellar heating can be reproduced in numerical simulations with an appropriate combination of massive halo black holes and giant molecular clouds. In addition to the proper amount of GMCs we used halo BHs of mass $M_{\mathrm{BH}}=$ $10^{7} M_{\odot}$. However, only half of the dark halo density $(\rho=$ $0.005 M_{\odot} / \mathrm{pc}^{3}$ ) or slightly less was needed in the form of black holes or other massive compact objects.

The strength of our conclusions was weakened by the fact that the observational data on the stellar disk heating is not very well constrained. The main difficulties lie in the determination of stellar ages which is a still rather complicated and error prone procedure.

This in mind we commenced a new study in order to explore consequences that the heating by massive halo objects and GMCs would have on the global properties of the stellar disk. Our main aim has been to explore observational constraints that might exclude the halo $\mathrm{BH}$ and GMC scenario from being possible source for the stellar disk heating. We have compared our simulation results to the observational data by Lewis \& Freeman (1989). Their work is the only one we know of in which the stellar velocity dispersion in the Galactic disk is measured at several different radii.

Our results indicate that the observed radial heating is not consistent with the scenarios in which the stellar disk heating is caused purely by the massive compact objects in the dark halo (we have called these objects black holes for brevity). In the simulations, the disk is typically heated in a manner which is inconsistent with observations in either the inner or outer disk. The radial scale lengths of stellar velocity dispersion from the simulations are typically much longer than those calculated from the observational data.

There is strong radial dependency in the GMC distribution. The Sun is located at the outer edge of the ring of molecular gas. The density maximum of the ring is located approximately at radius of $R \simeq 5 \mathrm{kpc}$. Most of the mass of the molecular gas is concentrated in the giant molecular clouds. The perturbational effect of the GMCs has to be taken into account with the massive compact halo objects.

Inclusion of the GMCs along with the BHs acts to bring the simulations more into line with the observational data. Nevertheless, the combined heating effect of the halo BHs and GMCs is still not consistent with the observational data in the case of $M_{\mathrm{BH}}=1 \times 10^{7} M_{\odot}$ : the radial scale length is still too long. However, the heating effect of the GMCs is relatively stronger if the BHs have mass only $M_{\mathrm{BH}}=1 \times 10^{6} M_{\odot}$. In this case the slope of the radial heating curve (see Fig. 9) is very close to the actual observations. The radial scale length is now about $16 \%$ larger than the value by Lewis \& Freeman and only slightly outside their formal error limits. We thus conclude that the latter scenario of the stellar disk heating can not be decisively ruled out by the observational evidence.

Acknowledgements. This work was supported by the Antares program of the Academy of Finland.

\section{References}

Barbanis, B., \& Woltjer, L. 1967, ApJ, 150, 461

Carlberg, R. 1987, ApJ, 322, 59

Carr, B. 1994, ARA\&A, 32, 531

Carr, B., \& Lacey, C. 1987, ApJ, 316, 23

Charlton, J., \& Laguna, P. 1995, ApJ, 444, 193

Flynn, C., \& Fuchs, B. 1994, MNRAS, 270, 471

Flynn, C., Sommer-Larsen, J., \& Christensen, P. 1996, MNRAS, 281, 1027

Friese, V., Fuchs, B., \& Wielen, R. 1995, in ed. P. C. van der Kruit, \& G. F. Gilmore, Stellar populations (Dordrecht: Kluwer), Proc. IAU Symp., 164, 414

Genzel, R. 1998, Nature, 391, 17

Hill, G. 1878, Am. J. Math., 1, 5

Hänninen, J., \& Flynn, C. 2002, MNRAS, 337, 731

Holmberg, J., \& Flynn, C. 2000, MNRAS, 313, 209

Jenkins, A., \& Binney, J. 1990, MNRAS, 245, 305

Jenkins, A. 1992, MNRAS, 257, 620

Julian W., \& Toomre, A. 1966, ApJ, 146, 810

Kuijken, K., \& Gilmore, G. 1989, MNRAS, 239, 651

Lacey, C., \& Ostriker, J. 1985, AJ, 299, 633

Lewis, J. R., \& Freeman, K. C. 1989, AJ, 97, 139

McLaughlin, D. E., Harris, W. E., \& Hanes, D. A. 1994, ApJ, 422, 486

Meylan, G., \& Heggie, D. C. 1997, A\&AR, 8, 1

Moore, B. 1993, ApJ, 413, L93

Moore, B., Ghigna, S., Governato, F., et al. 1999, ApJ, 524, L19

Narayan, C. A., \& Jog, J. A. 2002, A\&A, 394, 89

Press, W., Teukolsky, S., Vetterling, W., \& Flannery, B. 1992, Numerical Recipes in FORTRAN (Cambridge University Press)

Rubinstein, E., \& Bailyn, C. 1997, ApJ, 474, 701

Sanders, D. B., Solomon, P. M., \& Scoville, N. Z. ApJ, 276, 182

Scoville, N. Z., \& Sanders, D. B. 1986, The Galaxy and the Solar System, ed. R. Smoluchowski, et al., 69

Solomon, P. M., Sanders, D. B., \& Scoville, N. Z. 1979, The LargeScale Characteristics of the Galaxy, ed. W. B. Burton, 35

Spitzer, L., \& Schwarzschild, M. 1951, ApJ, 114, 385S

Spitzer, L., \& Schwarzschild, M. 1953, ApJ, 118, 106S

Velazquez, H., \& White, S. 1999, MNRAS, 304, 254

Wielen, R., Dettbarn, C., Fuchs, B., Jahreiß, H., \& Radons, G. 1992, The Stellar Populations of Galaxies, ed. B. Barbuy, \& A. Renzini, Proc. IAU Symp., 149, 81

Wisdom, J., \& Tremaine, S. 1988, AJ, 95, 925

Wouterloot, J. G. A., Band, J., Burton, W. B., \& Kwee, K. K. 1990, A\&A, 230, 21 